equation of a matrix has the same roots as its minimum equation and, when the latter is irreducible, the former is an exact power of the latter. But for all values of the $\xi_{i}$ in $F$ the quantities $x=\sum \xi_{i} u_{i}$ are in a division algebra and have irreducible minimum equation. Hence $R\left(\omega ; \xi_{1}, \cdots, \xi_{m}\right)=0$ is either irreducible in $F$ when the $\xi_{i}$ take on values in $F$ or is a power of an irreducible equation and is irreducible when it has no multiple roots. But the discriminant $D\left(\xi_{1}, \cdots, \xi_{m}\right)$ of $R\left(\omega ; \xi_{i}\right)$ is not identically zero, since $R\left(\omega ; \xi_{i}\right)$ is irreducible in $F\left(\xi_{1}, \cdots, \xi_{m}\right)$. Hence* there exists an infinity of values of the $\xi_{2}$ in $F$ for which $D \neq 0$ and $R=0$, of degree $n$, is the minimum equation of the corresponding quantities $x$.

The proof of Hilbert's theorem is non-algebraic and even for fields of algebraic numbers it would be desirable to have an algebraic proof of our important theorem on normal division algebras. The above furnishes such a proof. $\dagger$

Columbia University

\title{
A COMMUTATION RULE IN QUANTUM MECHANICS
}

\section{BY EUGENE FEENBERG}

In a recent paper $\mathrm{N}$. H. McCoy $\ddagger$ has developed general commutation rules for the algebra of the quantum mechanics of Born, Heisenberg and Jordan. It is the purpose of this note to point out a commutation rule which in part is implicit in McCoy's work.

The fundamental equation of quantum mechanics from which the algebra is developed is

* See Fricke, Algebra, vol. I, p. 96, for a rational proof of this result which holds for any non-modular field $F$.

$\dagger$ The author wishes to take this opportunity to announce a correction of the results of his two papers in this Bulletin, vol. 35 (1929), pp. 335-338, and in the Proceedings of the National Academy of Sciences, vol. 15 (1929), pp. 372-376, respectively. In both of these papers the Hilbert theorem was used and the results of these papers are correct only for fields for which a Hilbert irreducibility theorem is provable. In the statement of Hilbert's theorem in the paper in this Bulletin, the reading should be " $K$ any algebraic field over $R$, the field of all rational numbers," instead of " $K$ any infinite field."

$\ddagger$ Algebra of quantum mechanics, Transactions of this Society, vol. 31 (1929), pp. 793-806. 


$$
p q-q p=c I,
$$

in which the elements $p, q$ are interpreted as matrices or as noncommutative operators which multiply associatively. $I$ is the identity and $c$ a number, real or complex, but not zero. Únique inverse elements are assumed to exist and are written $q^{-1}, p^{-1}$. This assumption is not fulfilled for all elements $p, q$ satisfying equation (1), but by restricting exponents to positive values the results obtained are valid independent of the interpretation placed on $p, q$.

The result to be proved may be stated as follows:

$$
\begin{gathered}
\left(p^{i} q^{i}\right)^{m}\left(p^{i} q^{j}\right)^{n}\left(q^{k} p^{k}\right)^{r}\left(q^{l} p^{l}\right)^{s}=\left(q^{l} p^{l}\right)^{s}\left(q^{k} p^{k}\right)^{r}\left(p^{j} q^{j}\right)^{n}\left(p^{i} q^{i}\right)^{m} \\
i, j, k, l, m, n, r, s=0, \pm 1, \pm 2, \cdots
\end{gathered}
$$

By specializing the exponents it is seen that any pair of the terms $\left(p^{i} q^{i}\right)^{m},\left(p^{i} q^{i}\right)^{n},\left(q^{k} p^{k}\right)^{r},\left(q^{l} p^{l}\right)^{s}$ commute.

Proof. Since $p q=q p+c I$, we have

$$
(p q)(q p)=(q p)(p q), \quad\left(p^{-1} q^{-1}\right)\left(q^{-1} p^{-1}\right)=\left(q^{-1} p^{-1}\right)\left(p^{-1} q^{-1}\right) .
$$

Let us set $f=p q p^{-1} q^{-1}$. Then

$$
f q^{-1} p^{-1}=(p q)\left(p^{-1} q^{-1}\right)\left(q^{-1} p^{-1}\right)=(p q)\left(q^{-1} p^{-1}\right)\left(p^{-1} q^{-1}\right)=p^{-1} q^{-1},
$$

whence

$$
f=\left(p^{-1} q^{-1}\right)\left(q^{-1} p^{-1}\right)^{-1}=\left(p^{-1} q^{-1}\right)(p q) .
$$

It follows that

$$
(p q)\left(p^{-1} q^{-1}\right)=\left(p^{-1} q^{-1}\right) \cdot(p q), \quad(q p)\left(q^{-1} p^{-1}\right)=\left(q^{-1} p^{-1}\right)(q p) .
$$

If $G$ and $H$ are functions of $p, q$ commuting with $p q, q p, q^{-1} p^{-1}$, $p^{-1} q^{-1}, G H$ commutes with $p q, q p, q^{-1} p^{-1}, p^{-1} q^{-1}$. Since $(p q)^{-n}$ $=\left(q^{-1} p^{-1}\right)^{n}$ and $\left(p^{-1} q^{-1}\right)^{-n}=(q p)^{n}$ we find from (3) and (4) that

$$
(p q)^{m}(q p)^{n}=(q p)^{n}(p q)^{m}, \quad(n, m=0, \pm 1, \pm 2, \cdots) .
$$

To complete the proof we show that

$$
p^{n} q^{n}=\sum_{i=0}^{n} a_{i n}(p q)^{i}, \quad q^{n} p^{n}=\sum_{i=0}^{n} b_{i n}(q p)^{i},
$$

where $n=0,1,2, \cdots$, and the coefficients $a_{i n}, b_{i n}$ are numbers.

$$
p^{2} q^{2}=p(p q) q=p(q p+c I) q=(p q+c I) p q .
$$

It follows by induction that 
$p^{n} q^{n}=(p q+(n-1) c I) p^{n-1} q^{n-1}, \quad q^{n} p^{n}=(q p-(n-1) c I) q^{n-1} p^{n-1}$, $n=0,1,2, \cdots$, from which $(6)$ is readily proved. Then, by (5), we have

$$
\begin{aligned}
& \left(p^{n} q^{n}\right)\left(p^{m} q^{m}\right)=\left(p^{m} q^{m}\right)\left(p^{n} q^{n}\right) \\
& \left(p^{n} q^{n}\right)\left(q^{m} p^{m}\right)=\left(q^{m} p^{m}\right)\left(p^{n} q^{n}\right) \\
& \left(q^{n} p^{n}\right)\left(q^{m} p^{m}\right)=\left(q^{m} p^{m}\right)\left(q^{n} p^{n}\right), \quad(m, n=0,1,2, \cdots) .
\end{aligned}
$$

From (7) we obtain

$$
\begin{aligned}
\left(q^{-m} p^{-m}\right)\left(q^{-n} p^{-n}\right) & =\left(q^{-n} p^{-n}\right)\left(q^{-m} p^{-m}\right), \\
\left(q^{-m} p^{-m}\right)\left(p^{n} q^{n}\right) & =\left(p^{n} q^{n}\right)\left(q^{-m} p^{-m}\right),
\end{aligned}
$$

and four similar equations. Therefore (7) is true for $m, n=0$, $\pm 1, \pm 2, \cdots$, and since $\left(p^{i} q^{i}\right)^{-n}=\left(q^{-i} p^{-i}\right)^{n}$ we have the result which was to be proved:

$\left(p^{i} q^{i}\right)^{m}\left(p^{j} q^{j}\right)^{n}=\left(p^{j} q^{j}\right)^{n}\left(p^{i} q^{i}\right)^{m}$, $\left(p^{i} q^{i}\right)^{m}\left(q^{j} p^{j}\right)^{n}=\left(q^{j} p^{j}\right)^{n}\left(p^{i} q^{i}\right)^{m}$, $\left(q^{i} p^{i}\right)^{n}\left(q^{j} p^{j}\right)^{n}=\left(q^{j} p^{j}\right)^{n}\left(q^{i} p^{i}\right)^{m}, \quad(i, j, m, n=0, \pm 1, \pm 2, \cdots)$.

By specializing a general identity McCoy finds

$$
q^{m} p^{m^{\prime}} q^{n} f^{n^{\prime}}=q^{n^{\prime}} p^{n} q^{m^{\prime}} p^{m}, \quad m+n=m^{\prime}+n^{\prime} .
$$

To illustrate the use of the theorem we verify this identity:

$$
\begin{aligned}
q^{m} p^{m^{\prime}} q^{n} p^{n^{\prime}} & =\left(q^{m} p^{m}\right)\left(p^{m^{\prime}-m} q^{m^{\prime}-m}\right)\left(q^{n^{\prime}} p^{n^{\prime}}\right) \\
& =\left(q^{n^{\prime}} p^{n^{\prime}}\right)\left(p^{m^{\prime}-m} q^{m^{\prime}-m}\right)\left(q^{m} p^{m}\right)=q^{n^{\prime}} p^{n} q^{m^{\prime}} p^{m} .
\end{aligned}
$$

If inverses do not exist and $m^{\prime}<m$

$$
\begin{aligned}
q^{m} p^{m^{\prime}} q^{n} p^{n^{\prime}} & =q^{m-m^{\prime}}\left(q^{m^{\prime}} p^{m^{\prime}}\right)\left(q^{n} p^{n}\right) p^{n^{\prime}-n} \\
& =q^{m-m^{\prime}}\left(q^{n} p^{n}\right)\left(q^{m^{\prime}} p^{m^{\prime}}\right) p^{n^{\prime}-n}=q^{n^{\prime}} p^{n} q^{m^{\prime}} p^{m} .
\end{aligned}
$$

Harvard University 